\title{
Polyhydroxy surfactants for the formulation of lipid nanoparticles (SLN and NLC): Effects on size, physical stability and particle matrix structure
}

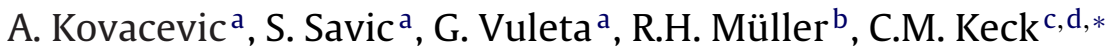 \\ a Department of Pharmaceutical Technology and Cosmetology, Faculty of Pharmacy, University of Belgrade, VojvodeStepe 450, 11221 Belgrade, Serbia \\ ${ }^{\mathrm{b}}$ Department of Pharmaceutics, Biopharmaceutics \& NutriCosmetics, Freie Universität Berlin, Kelchstr. 31, 12169 Berlin, Germany \\ ${ }^{\mathrm{c}}$ Department of Applied Logistics and Polymer Sciences, University of Applied Sciences Kaiserslautern, Pirmasens, Germany \\ ${ }^{\mathrm{d}}$ Institute of Biosciences (IBS), University Putra Malaysia (UPM), Serdang-Kuala Lumpur, Malaysia
}

\section{A R T I C L E I N F O}

\section{Article history:}

Received 15 September 2010

Received in revised form

21 December 2010

Accepted 27 December 2010

Available online 8 January 2011

\section{Keywords:}

Polyhydroxy surfactants

Solid lipid nanoparticles

SLN

Nanostructured lipid carriers

NLC

Physical stability

Crystallinity

\begin{abstract}
A B S T R A C T
The two polyhydroxy surfactants polyglycerol 6-distearate (Plurol ${ }^{\circledR}$ Stearique WL1009 - (PS)) and caprylyl/capryl glucoside (Plantacare ${ }^{\circledR} 810-(\mathrm{PL})$ ) are a class of PEG-free stabilizers, made from renewable resources. They were investigated for stabilization of aqueous solid lipid nanoparticle (SLN) and nanostructured lipid carrier (NLC) dispersions. Production was performed by high pressure homogenization, analysis by photon correlation spectroscopy (PCS), laser diffraction (LD), zeta potential measurements and differential scanning calorimetry (DSC). Particles were made from Cutina CP as solid lipid only (SLN) and its blends with Miglyol 812 (NLC, the blends containing increasing amounts of oil from 20\% to 60\%). The obtained particle sizes were identical for both surfactants, about $200 \mathrm{~nm}$ with polydispersity indices below 0.20 (PCS), and unimodal size distribution (LD). All dispersions with both surfactants were physically stable for 3 months at room temperature, but Plantacare (PL) showing a superior stability. The melting behaviour and crystallinity of bulk lipids/lipid blends were compared to the nanoparticles. Both were lower for the nanoparticles. The crystallinity of dispersions stabilized with PS was higher, the zeta potential decreased with storage time associated with this higher crystallinity, and leading to a few, but negligible larger particles. The lower crystallinity particles stabilized with PL remained unchanged in zeta potential (about $-50 \mathrm{mV}$ ) and in size. These data show that surfactants have a distinct influence on the particle matrix struture (and related stability and drug loading), to which too little attention was given by now. Despite being from the same surfactant class, the differences on the structure are pronounced. They are attributed to the hydrophobic-lipophilic tail structure with one-point anchoring in the interface (PL), and the loop conformation of PS with two hydrophobic anchor points, i.e. their molecular structure and its interaction with the matrix surface and matrix bulk. Analysis of the effects of the surfactants on the particle matrix structure could potentially be used to further optimization of stability, drug loading and may be drug release.
\end{abstract}

(c) 2011 Elsevier B.V. All rights reserved.

\section{Introduction}

Lipid nanoparticles were developed in the last decade of the last century as alternative carrier system to emulsions, liposomes and polymeric nanoparticles (Lucks and Müller, 1996; Mehnert and Mäder, 2001; Müller et al., 2000a,b, 1995). Solid lipid nanoparticles (SLN) and nanostructured lipid carriers (NLC) are the two main types of lipid nanoparticles. The research activities in SLN and NLC in the last two decades focussed mainly on pharmaceutical nondermal administration routes, i.e. parenteral (Blasi et al., 2007; Joshi

\footnotetext{
* Corresponding author at: Department of Applied Logistics and Polymer Sciences, University of Applied Sciences Kaiserslautern, Pirmasens, Germany. Tel.: +49 (0)63312483 20; fax: +49 (0)6331248344.

E-mail addresses: ck@ckc-berlin.de, conny-keck@web.de (C.M. Keck).
}

and Müller, 2009; Wissing et al., 2004), peroral (Muchow et al., 2008; Müller et al., 2006; Sarmento et al., 2007), ocular (Attama et al., 2007; Ugazio et al., 2002) and pulmonary (Liu et al., 2008) administration. During the last 5 years SLN and NLC have been intensively investigated for dermal application because of many positive features that have been reported after their application to the skin (Müller et al., 2007, 2002b). Due to the lipid matrix, the small particle size and related adhesive properties, the residence time of SLN and NLC on the skin is prolonged. There is a lipid interaction of the particle matrix with skin surface lipids, affecting drug absorption (Lombardi Borgia et al., 2007; Santos Maia et al., 2002). Occlusive properties, increase in skin hydration, modified release of actives, targeting effects to specific skin strata - especially when the drug is located at the particle surface - are also positive features of lipid nanoparticles (Müller et al., 2007). SLN were found to enhance the skin penetration of several dermally applied drugs 
(Lombardi Borgia et al., 2005; Souto et al., 2004; Stecova et al., 2007). The imperfections and fluid domains in the lipid matrix of NLC show a higher loading capacity for a number of drugs than SLN and avoid/minimize potential expulsion of drug during the storage (Mehnert and Mäder, 2001; Pardeike et al., 2009).

Due to the production from physiological and biodegradable lipids, SLN and NLC exhibit good skin tolerability. Good skin tolerability requires also the use of well tolerated surfactants or polymeric stabilizers. A variety of different stabilizers have been used up to now for lipid nanoparticles including ionic (e.g. sodium cholate), non-ionic (e.g. polysorbates), amphoteric surfactants (phospholipids), and polymeric stabilizers (e.g. poloxamers, polyvinyl alcohols) (Siekmann, 1994; Siekmann and Westesen, 1994a,b). Polymeric stabilizers are particularly advisable for dermal application since they are less likely to penetrate beyond the stratum corneum and hence, they are less likely to cause skin irritation. However, the use of polymers as only stabilizers sometimes requires relatively high amounts for effective stabilization of lipid nanoparticles (Siekmann, 1994; Siekmann and Westesen, 1994a). For example, Siekmann and Westesen (1994a) reported that $2 \%$ $(\mathrm{w} / \mathrm{w})$ Tyloxapol ${ }^{\circledR}$ were insufficient to stabilize a $10 \%(\mathrm{w} / \mathrm{w})$ tripalmitine lipid nanoparticles dispersion.

Non-ionic surfactants being uncharged have a low skin sensitization potential and therefore they are preferentially used for dermal application. Two main groups of non-ionic surfactants are the ethylene oxide-based surfactants with polyethylene glycol (PEG) blocks and the polyhydroxy (polyol based) surfactants, having a polyhydroxy head group chain. Nowadays cosmetic industry moves away from ethoxylated surfactants, PEG-free is becoming a quality criterion (Cosmetic Ingredient Reviews, 1999; Johnson, 2001; Lanigan and Yamarik, 2001; Tadros, 2005). In addition, with the increasing awareness of environmental issues the cosmetic and pharmaceutical industry has a trend to use excipients made from renewable materials. In this sense, there is growing interest in polyhydroxy surfactants as the polyhydroxy head group can be derived from a naturally occurring carbohydrate. Polyhydroxy surfactants have been developed substituting ethylene oxide by hydroxy groups. Linking the hydrophilic and hydrophobic parts in different ways affects HLB and stabilizing properties of these molecules.

Typical hydrophilic building blocks in their structure are glycerol, carbohydrates and their alcohols (sucrose, glucose, sorbitol) and glycols. The main categories of polyhydroxy surfactants are: glycolesters, glycerol (and polyglycerol) esters, glucosides (and polyglucosides), sucrose and sorbitan esters.

Polyhydroxy surfactants have the properties of showing stronger lipophilicity and hydrophilicity compared to ethoxylated surfactants. The stronger hydrophilicity results from the hydroxy groups in the structure providing stronger hydrogen bonds with water compared to the oxygen in the PEG. Consequently at high temperature dehydration of the hydrophilic head group can be prevented. Thus, they have a higher critical flocculation temperature. Fatty acid or alcohol chains as hydrophobic part of the molecule are more hydrophobic than the propylene oxide chain in e.g. PEG-containing poloxamers. In addition, these chains are able to anchor in the surface of oil droplets or lipid nanoparticles. Additional reasons for the increasing interest in these molecules can be their minor susceptability to $\mathrm{pH}$ changes and electrolyte presence compared to ethoxylated stabilizers (Söderman and Johansson, 1999). Polyhydroxy surfactants possess therefore favourable technological and dermatological properties, which is why they are meanwhile often used in dermal formulations. Therefore they were investigated in this study for their ability to stabilize lipid nanoparticles, both SLN and NLC.

Besides the physical stability, the stabilizers can have effects on the crystallinity and the kinetics of polymorphic transitions of lipid after crystallization of nanoparticles (Garti and Yano, 2001). Previous investigations on tripalmitin nanoparticles indicated that rigid stabilizer chains are needed to induce crystallization of tripalmitin at higher temperatures (Bunjes et al., 2002). The influence of stabilizers onto the crystallization is particularly important in the formulation of lipid nanoparticles made from low melting lipids. These nanocarriers can be interesting for the processing and the formulation of temperature-sensitive actives such as peptides and proteins, excluding the use of high melting lipids. Additionally, studies about the effect of stabilizers on the crystallization process and crystalline structure of the resulting particle matrix may provide insight into the interaction of the dispersed lipid and the stabilizer used (Souto, 2005).

The first aim of the present study was to evaluate the ability of polyhydroxy surfactants to create small sized lipid nanoparticle dispersions with narrow size distribution. Two surfactants with basically different structures having glycerol and glucose as hydrophilic groups (polyglycerol versus polyglucoside chain), were used in this study (Table 1). Particle formation was systematically studied using cetyl palmitate as solid lipid only (SLN) and also using mixtures of this solid lipid with increasing amounts of Miglyol 812 (NLC). Secondly, the influence of the surfactant onto the physical stability and the crystalline state of the particle matrix was investigated. This should provide information about the stabilizing ability, and to which extent the two different surfactants influence the lipid structure of the particles, in comparison to each other and to bulk lipid.

\section{Materials and methods}

\subsection{Materials}

For the production of the lipid nanoparticles, the solid lipid cetyl palmitate (Cutina ${ }^{\circledR} \mathrm{CP}$ ) was kindly provided by Cognis (Düsseldorf, Germany). The medium chain triglyceride oil (Miglyol ${ }^{\circledR}$ 812) was purchased from Caelo GmbH (Hilden, Germany). Polyglycerol 6-distearate(Plurol ${ }^{\circledR}$ Stearique WL1009 (PS)) was kindly provided by Gattefosee (Weil am Rhein, Germany) and caprylyl/capryl glucoside(Plantacare ${ }^{\circledR} 810$ (PC)) by Cognis (Düsseldorf, Germany).The purified water was obtained by reverse osmosis from a Milli Q Plus, Millipore system (Schwalbach, Germany). All chemicals were used directly as received without further purification.

\subsection{Methods}

\subsubsection{Characterization of the bulk lipid material}

Differential scanning calorimetry (DSC) was employed to investigate the effect on the crystalline structure after inclusion of oil into the solid lipid. In order to mimic the production conditions of NLC, the bulk mixtures of solid lipid and oil at different ratios (Table 2) were heated up to $75^{\circ} \mathrm{C}$, kept at that temperature for $1 \mathrm{~h}$ and subsequently cooled to room temperature. This imitates the production process of lipid nanoparticles by homogenization in the melted condition, and the subsequent cooling and recrystallization. These tempered lipid blends were then investigated using a Mettler DSC 821e apparatus (Mettler Toledo; Gießen, Switzerland) using accurately weighed samples of 1-2 mg.

\subsubsection{Preparation of lipid nanoparticles}

The total amount of lipid phase (solid lipid, oil) was kept constant in all lipid nanoparticle suspensions (10\%, w/w). SLN contained solid lipid only (Cutina CP) while in NLC a portion of the solid lipid has been replaced by oil (Miglyol ${ }^{\circledR} 812$ ). The lipid nanoparticles in suspension were stabilized using $1 \%(\mathrm{w} / \mathrm{w})$ of polyhydroxy surfactants, either PS or PL. Table 3 provides the composition of 
Table 1

Trade names, chemical names and struture of polyhydroxy surfactants used for the preparation of the lipid nanoparticle dispersions.

\begin{tabular}{|c|c|}
\hline Trade name of surfactant & $\begin{array}{l}\text { Structural formula and chemical name of } \\
\text { surfactant }\end{array}$ \\
\hline $\begin{array}{l}\text { Plurol Stearique }{ }^{\circledR} \text { WL } 1009 \text { (PS) } \\
H L B=9-10\end{array}$ & $\mathrm{C}_{17} \mathrm{H}_{35}$ \\
\hline
\end{tabular}

Table 2

DSC parameters of the tempered bulk solid lipid and bulk lipid blends with increasing oil content.

\begin{tabular}{|c|c|c|c|c|}
\hline Lipid & Melting temperature $\left[{ }^{\circ} \mathrm{C}\right]$ & Onset temperature $\left[{ }^{\circ} \mathrm{C}\right]$ & Enthalpy $[\mathrm{J} / \mathrm{g}]$ & Integral (mJ) \\
\hline Cutina ${ }^{\circledR} \mathrm{CP}$ (bulk lipid) & 52.44 & 47.89 & 220.46 & 296.52 \\
\hline $80 \%$ Cutina ${ }^{\circledR} \mathrm{CP}+20 \%$ Miglyol $^{\circledR} 812$ & 48.70 & 39.88 & 124.29 & 202.09 \\
\hline $70 \%$ Cutina $^{\circledR} \mathrm{CP}+30 \%$ Miglyol $^{\circledR} 812$ & 47.72 & 38.07 & 91.11 & 164.82 \\
\hline $60 \%$ Cutina $^{\circledR} \mathrm{CP}+40 \%$ Miglyol $^{\circledR} 812$ & 46.90 & 36.68 & 69.87 & 123.95 \\
\hline $50 \%$ Cutina ${ }^{\circledR} \mathrm{CP}+50 \%$ Miglyol $^{\circledR} 812$ & 46.07 & 35.54 & 55.80 & 116.30 \\
\hline $40 \%$ Cutina $^{\circledR} \mathrm{CP}+60 \%$ Miglyol $^{\circledR} 812$ & 45.28 & 33.80 & 55.90 & 103.87 \\
\hline
\end{tabular}

the formulations, from SLN (=100\% solid lipid) and the NLC with increasing oil content in the lipid blend from 20\% Miglyol 812 to $60 \%$.

Preparation of the lipid nanoparticle suspensions was carried out by hot high pressure homogenization ( $\mathrm{HPH}$ ). Briefly, the lipid phase was melted at $75^{\circ} \mathrm{C}$ and subsequently dispersed by high speed stirring ( $8000 \mathrm{rpm}$ for $1 \mathrm{~min}$ ) in the hot aqueous surfactant solution of identical temperature. The dispersing was performed using an Ultra-Turrax (Janke \& Kunkel, Staufen, Germany). The obtained pre-emulsion was then subjected to HPH using a Micron LAB 40 (APV Deutschland GmbH, Germany). Five homogenization cycles at 500 bar and a temperature of $75^{\circ} \mathrm{C}$ were applied. After $\mathrm{HPH}$ the obtained hot o/w nanoemulsion was filled in silanized transparent glass vials (glass quality II), which were immediately sealed. Despite using glass quality II, the vials were siliconized to further minimize the adsorption of nanoparticles onto the walls. This adsorption is known to promote aggregation by fusion of adsorbed nanoparticles. The vials were placed into a water bath adjusted to $20^{\circ} \mathrm{C}$ to control the cooling rate of the nanoemulsions and the velocity of crystallization. To investigate the physical stability, the lipid dispersions were stored at $25 \pm 2{ }^{\circ} \mathrm{C}$ for a period of 90 days. The samples were analyzed at previously determined time intervals (day 0 , 1 st, 30th and 90th day).

\subsubsection{Particle size analysis}

Particle size analysis was performed by dynamic light scattering (DLS), also known as photon correlation spectroscopy (PCS), using a Malvern Zetasizer Nano ZS (Malvern Instruments, UK). Prior to the measurements all samples were diluted using ultra-purified water to yield a suitable scattering intensity. DLS data were analyzed at $25^{\circ} \mathrm{C}$ using the general purpose mode. DLS yields the hydrodynamic diameter (intensity weighted mean diameter, $z$-average,

Table 3

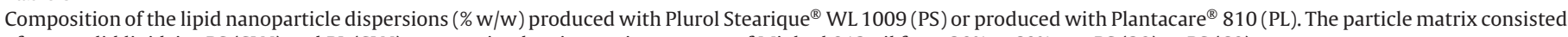
of pure solid lipid. i.e. PS (SLN) and PL (SLN), or contained an increasing amount of Miglyol 812 oil from 20\% to 60\%. e.g. PS (20) to PS (60).

\begin{tabular}{|c|c|c|c|c|c|}
\hline Formulation & Cutina $^{\circledR} \mathrm{CP}$ & Miglyol ${ }^{\circledR} 812$ & Plurol Stearique ${ }^{\circledR}$ WL 1009 & Plantacare ${ }^{\circledR} 810$ & Purified water up to \\
\hline PS (SLN) & 10.0 & - & 1.0 & - & 100.0 \\
\hline PS (20) & 8.0 & 2.0 & 1.0 & - & 100.0 \\
\hline PS (30) & 7.0 & 3.0 & 1.0 & - & 100.0 \\
\hline PS (40) & 6.0 & 4.0 & 1.0 & - & 100.0 \\
\hline PS (50) & 5.0 & 5.0 & 1.0 & - & 100.0 \\
\hline PS (60) & 4.0 & 6.0 & 1.0 & - & 100.0 \\
\hline PL (SLN) & 10.0 & - & - & 1.0 & 100.0 \\
\hline PL (20) & 8.0 & 2.0 & - & 1.0 & 100.0 \\
\hline PL (30) & 7.0 & 3.0 & - & 1.0 & 100.0 \\
\hline $\mathrm{PL}(40)$ & 6.0 & 4.0 & - & 1.0 & 100.0 \\
\hline PL (50) & 5.0 & 5.0 & - & 1.0 & 100.0 \\
\hline PL (60) & 4.0 & 6.0 & - & 1.0 & 100.0 \\
\hline
\end{tabular}


$z$-ave) and the polydispersity index (PI) as a measure of the width of the particle size distribution. The PI ranges from 0 to 1 while monodisperse populations yield theoretically a PI of 0 . Very narrow distributed particle populations possess PI values of about $0.02-0.05$, o/w emulsions for parenteral nutrition by intravenous infusion typically from 0.10 to 0.25 . The $z$-average and PI of the investigated samples were obtained by calculating the average of 10 measurements at an angle of $173^{\circ}$ in $10 \mathrm{~mm}$ diameter disposable plastic cells.

The measuring range of the Zetasizer is from approximately $6 \mathrm{~nm}$ to $6 \mu \mathrm{m}$. Therefore, in order to detect potential larger particles and oil droplets static light scattering (SLS), also known as laser diffractometry (LD), with a measuring range up to $2000 \mu \mathrm{m}$ was applied as additional characterization method using a Malvern Mastersizer 2000 (Malvern Instruments, UK). SLS data were analyzed using the Mie theory with the optical parameters 1.456 (real refractive index) and 0.01 (imaginary refractive index). The SLS data yield a volume distribution. The volume weighted diameters $d(v) 50 \%, d(v) 90 \%$ and $d(v) 99 \%$ were used to characterize the dispersions. The diameter values indicate the percentage of particles possessing a diameter equal or lower than the given values (Rawle). For example, $d(v)$ 99\% means that $99 \%$ of the volume of the particles is below the given size. Sonification prior and during the measurement was not performed to avoid the destruction of possible aggregates within the sample. Such aggregates are a sensitive marker for insufficient stabilization, and will mainly affect the diameters $90 \%$ and $99 \%$.

\subsubsection{Zeta potential analysis}

The zeta potential was determined by the measurement of the electrophoretic mobility using a Malvern Zetasizer Nano ZS (Malvern Instruments, UK). The field strength applied was $20 \mathrm{~V} / \mathrm{cm}$. The conversion into the zeta potential was performed using the Helmoltz-Smoluchowski equation:

$\zeta=\mathrm{EM} \times \frac{4 \pi \eta}{\varepsilon}$

where $\zeta$ is zeta potential, EM the electrophoretic mobility, $\eta$ the viscosity of the dispersion medium and the dielectric constant $\varepsilon$. To avoid the fluctuation in the zeta potential due to variations in the conductivity of purified water, which can range from 1 to $10 \mu \mathrm{S} / \mathrm{cm}$, the conductivity of the water was adjusted to $50 \mu \mathrm{S} / \mathrm{cm}$ using $0.9 \%$ $(\mathrm{w} / \mathrm{v})$ sodium chloride solution. The $\mathrm{pH}$ during the measurements was in the range of 5.5-6.0. Each sample was measured three times and mean value and standard deviation (SD) are presented.

\subsubsection{Differential scanning calorimetry investigations of the lipid nanoparticle suspensions}

Thermal analysis was performed using again the Mettler DSC 821e apparatus (Mettler Toledo, Gieben, Switzerland). The amount of the sample (aqueous dispersion) was calculated this way, that it contained approx. 1-2 mg lipid (accurately weighted). For a 10\% NLC dispersion this is approx. $10-20 \mu \mathrm{l}$, which were placed in $40 \mu \mathrm{l}$ aluminum pans. The heating runs were performed from 20 to $90^{\circ} \mathrm{C}$ at a heating rate of $10 \mathrm{~K} \mathrm{~min}^{-1}$. An empty aluminum pan was used as reference. Onset temperature, melting point, enthalpy and integral were calculated by the STAR ${ }^{\mathrm{e}}$ Software(Mettler Toledo, Switzerland). Furthermore, the recrystallization index (RI) i.e. percentage of re-crystallized solid lipid related to initial solid lipid concentration (not total lipid concentration of solid and liquid lipid!) was calculated using the following equation (Freitas and Müller, 1999):

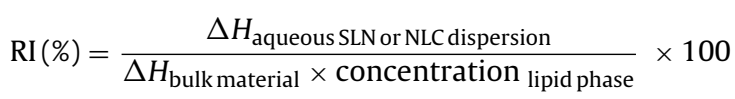

where $\Delta H_{\text {aqueous SLN or NLC dispersion }}$ and $\Delta H_{\text {bulk material }}$ are the melting enthalpy $(\mathrm{J} / \mathrm{g})$ of SLN and NLC dispersion and bulk material, respectively. The concentration of the lipid phase is given in parts solid lipid in the total suspension (e.g. $10 \%$ suspension is $10 / 100$ parts $=0.1$, a $10 \%$ NLC suspension composed of $8 \%$ solid lipid and $2 \%$ oil is $=0.08$ ). In case the RI of the tempered bulk material is calculated, the concentration is $=1$.

\subsubsection{Light microscopy}

SLS measurements can overlook a small number of larger particles besides a small-sized main particle population (Keck and Müller, 2008). Therefore to detect potential instability and aggregates during storage, the dispersions were additionally monitored using a light microscope (Orthoplan Leitz, Wetzlar, Germany). The microscope was equipped with a CMEX 3200 digital camera (Euromex microscopes, Arnheim, Netherlands) connected to the Image Focus software version 1.3.1.4. Microscopic pictures were taken from the undiluted lipid nanoparticle dispersions to increase the probability of detecting even a few large aggregates. Aim was not to analyze the nanoparticles themselves, because they are below the detection limit (about $500 \mathrm{~nm}$ ) of the microscope. Magnifications applied were $160,400,630$ and 1000 fold.

\section{Results and discussion}

\subsection{Characterization of bulk lipid material}

A DSC study was performed to understand the blending behaviour of the solid lipid Cutina $\mathrm{CP}$ with increasing amounts of oil, i.e. Miglyol 812. It should provide information about the influence of the oil onto the melting behaviour and change in crystallinity to obtain information on the inclusion of the oil into the bulk of solid lipid. The thermo-analytical parameters as derived from the DSC measurements are given in Table 2. Cetyl palmitate revealed two peaks at $38.94{ }^{\circ} \mathrm{C}$ (small peak) and $52.42^{\circ} \mathrm{C}$ (main peak) (Fig. 1). Based on literature data, the first peak with lower melting point is attributed to the $\alpha$-polymorphic form (thermodynamic instable modification) whereas the second peak belongs to the $\beta$-polymorphic form (stable modification) (Saupe et al., 2005).

Cetyl palmitate and medium chain triglycerides exhibit a good miscibility and thus a homogeneous lipid matrix should be obtained in the NLC. The DSC thermograms show that above $20 \%$ oil, the separate peak of the $\alpha$-modification disappears (Fig. 1, middle), at the same time the peak broadens (increase in difference between melting temperature and onset) and the peak height (also area under the curve) decreases, hence the crystallinity is reduced (Fig. 1, middle and lower). This supports that the oil is molecularly dispersed in the lipid blend, at least at lower concentrations, which creates distortion in the lipid matrix.

A melting point depression occurs, if a second compound is dissolved in a first compound. Therefore the melting point determination is also used for purity analysis of materials. With increasing concentration of the second compound, the melting point decreases in a linear fashion. A decreasing melting point, and onset temperature, was observed with increasing oil content in the lipid blends (Table 2). The decrease was linear up to $60 \%$ oil in the lipid blend, indicating good miscibility.

With $20 \%(\mathrm{w} / \mathrm{w})$ of oil the difference between the melting and the onset temperatures was $8.82^{\circ} \mathrm{C}$ and increased to $11.48^{\circ} \mathrm{C}$ when the blends contained $60 \%(\mathrm{w} / \mathrm{w})$ of the oil. Therefore, for the given concentration range the depression of the onset temperatures did not occur in a linear fashion. There is a strong decay up to $20 \%$ and $30 \%$ oil content, then the decay flattens with increasing contents of oil (Fig. 2). The broadening of the peak is caused by a more complex, 

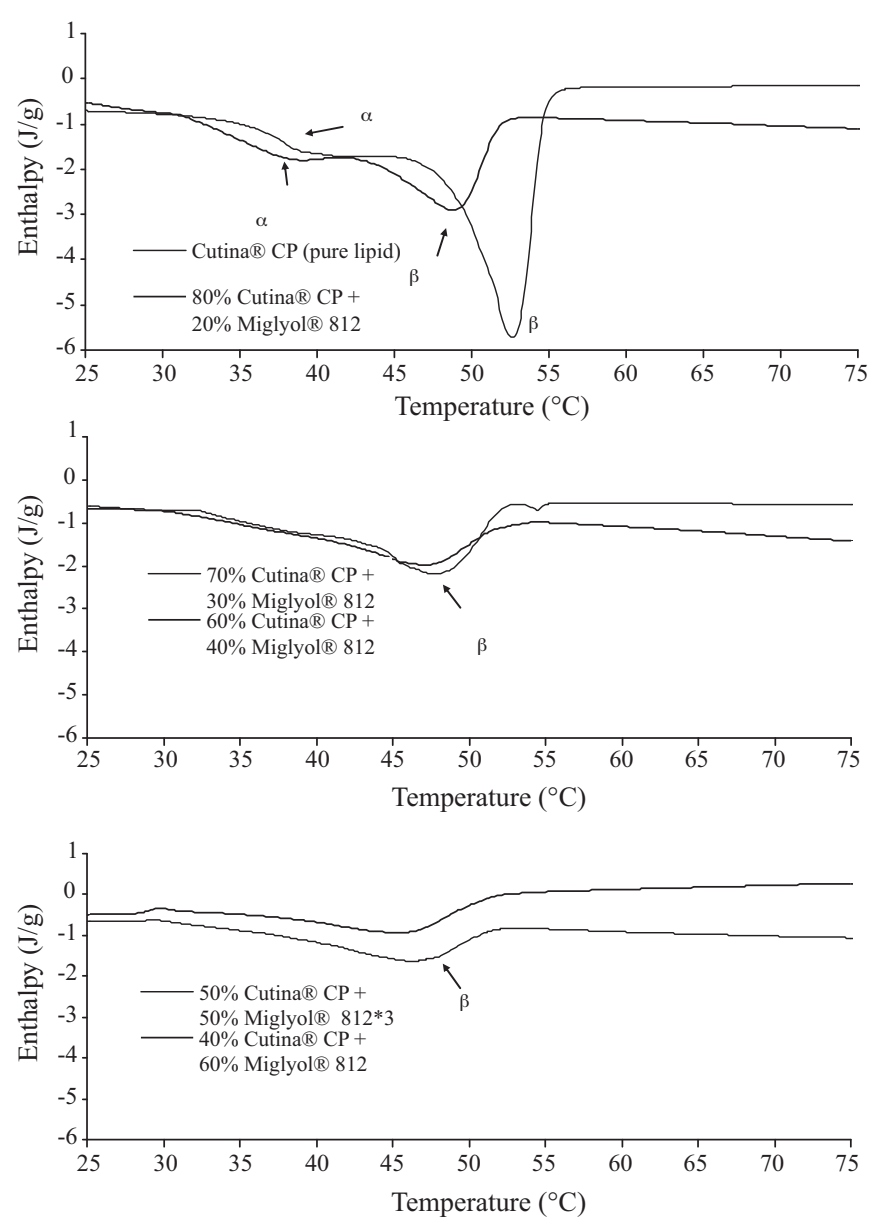

Fig. 1. DSC heating thermograms of tempered bulk solid lipid Cutina CP and tempered lipid blends with increasing Miglyol 812 content from $20 \%$ to $60 \%$.

distorted structure of the lattice, the flattening of the onset temperature decay might indicate, that one approaches the maximum solubility of the oil in the solid lipid.

Distortion of the lattice and reduced crystallinity is clearly seen by the decrease in melting enthalpy of the blends with increasing oil content compared to the pure solid lipid. The enthalpy decreases from about $220 \mathrm{~J} / \mathrm{g}$ to $55 \mathrm{~J} / \mathrm{g}$ at $50 \%$ oil content in the blend, accompanied by a decrease in crystallinity from $100 \%$ to $25.3 \%$ (Table 2 ).

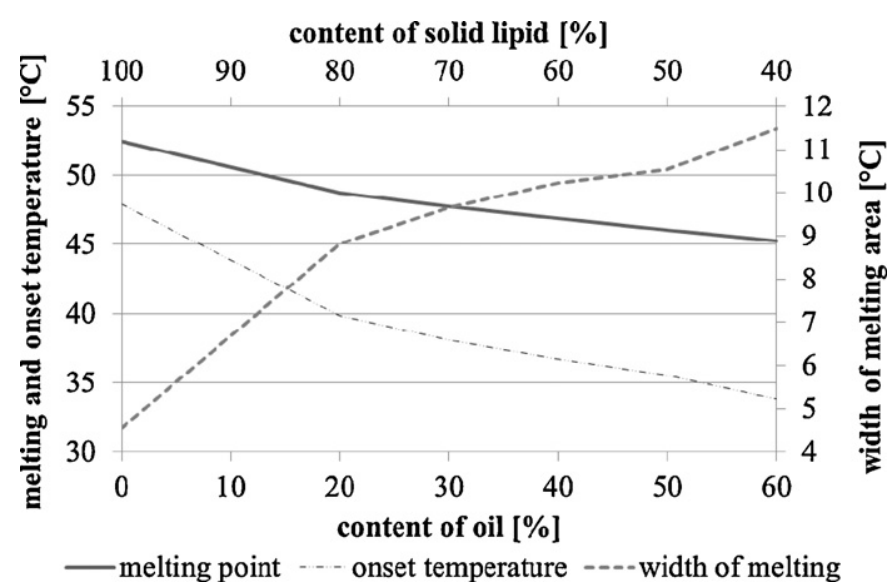

Fig. 2. Decrease of melting temperature and onset temperature, and increase of width betweeen these two temperatures at increasing oil content of the bulk lipids from $0 \%$ to $60 \%$, as deteremined by DSC (data from Table 1 ).
Interestingly, the crystallinity does not further decrease when moving from $50 \%$ oil to $60 \%$ oil, it stays unchanged at $25.3 \%$. This can also seen as indication that one approaches the solubility limited of the oil in the blend.

According to Müller et al. at low oil concentration, the oil molecules are distributed within the solid lipid matrix and the imperfect type of NLC is obtained (Müller et al., 2002a). In case the oil exceeds its solubility in the solid lipid, the multiple type of NLC is formed. The oil precipitates separately e.g. in liquid nanocompartments within the lipid particle matrix. This occurs during the cooling process. At high temperatures, complete miscibility occurs between the melted solid lipid and oil. During the cooling down process the solubility of the oil in the solid lipid is exceeded. Therefore, due to the miscibility gap phase separation occurs. The oil precipitates in the form of fine droplets being incorporated into the solid lipid matrix during the cooling process of NLC. Based on the DSC bulk data, a phase separation cannot be excluded when producing NLC with the high oil concentrations, especially because crystallization processes in the nanodimension differ from bulk crystallisation. Examples are the reduction of the melting temperature (Gibbs-Thomson effect (Perez, 2005)) or the lack of re-crystallization by formation of supercooled nanoparticles.

There is a kind of controverse discussion, where the phase separated oil is located. Müller et al. (2002a) and zur Mühlen and Mehnert suggest location in form of nanocompartments throughout the solid lipid matrix (original model). However, the nanocompartments might be enriched in the core or the shell (Saupe et al., 2005) or located at the surface (oil layer on surface?). There are also spoon-like structures suggested (Jores et al., 2005, 2004). It is outside the scope of this article to discuss in detail the physics behind the various theories. At least it can be stated, that the location will definitely differ depending on the chemical nature of solid and liquid lipid, the solubility/miscibility of lipids in each other, production conditions, and interfacial tensions between lipid and water phase (interfacial energies). As conclusion, the described structures could exist besides each other, depending on the lipids used.

\subsection{Particle size analysis and physical stability}

Table 3 gives an overview of the formulations produced. The total amount of lipid phase was kept constant $(10 \%, w / w)$, the percentage of oil in the lipid matrix of the NLC was increased from $20 \%$ to $60 \%$, according to the investigated bulk lipids. The particle sizes for all formulations have been evaluated by DLS and SLS immediately after production and during a storage period of up to 90 days.

Under optimized production conditions (500 bar, five homogenization cycles, $75^{\circ} \mathrm{C}$ ) small lipid nanoparticles with a relatively uniform size distribution were obtained. Figs. 3 and 4 show the obtained results. The mean DLS particle diameters were around or below $200 \mathrm{~nm}$ with PI values below 0.2 indicating monomodal size distributions. During the observation period of 90 days the mean diameters and PI values did practically not change. This indicates good physical stability of the lipid nanoparticles stabilized with both polyhydroxy surfactants.

Cetyl palmitate is a wax produced by catalytic esterification of fatty alcohol (cetyl alcohol) and fatty acids (palmitic acid) (Saupe et al., 2005). It was suggested that surface active partial glycerides (mono-, and diglycerides and their mixtures) facilitate emulsification and form more rigid surfactant films around the lipid nanoparticles and thus improve long term physical stability (Bunjes et al., 2003; Westesen et al., 1993). However, for a relatively pure cetyl palmitate as used in this study, primarily the surfactant used is responsible for physically stable lipid nanoparticles (Saupe et al., 

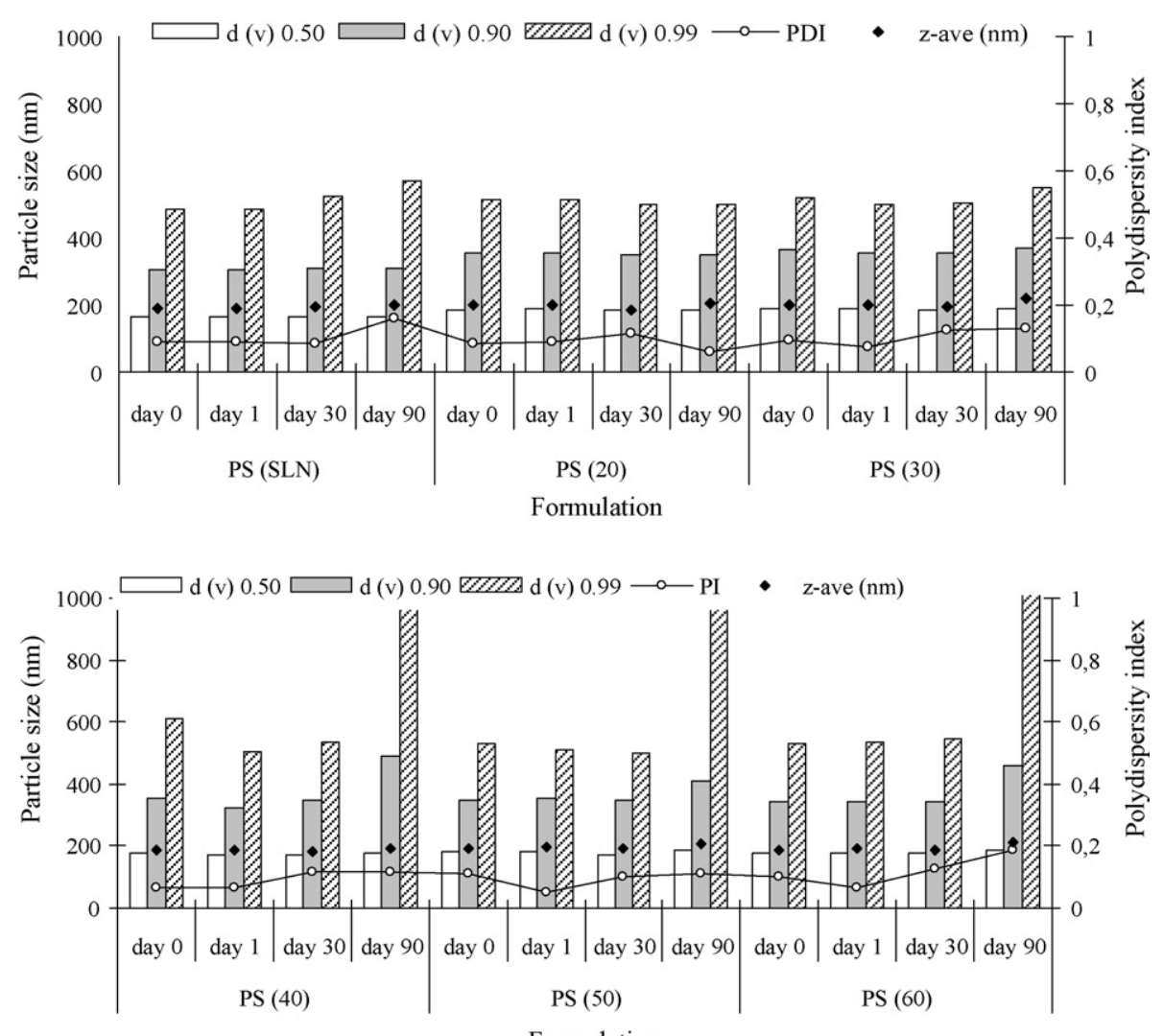

Fig. 3. PCS data (diameter and PI) and LD data (diameters $d(v) 50 \%, d(v) 90 \%$ and $d(v) 99 \%)$ of the formulations stabilized with PS (polyglyceryl 6-distearate) plotted as function of time $(0,1,30,90$ days $)$.

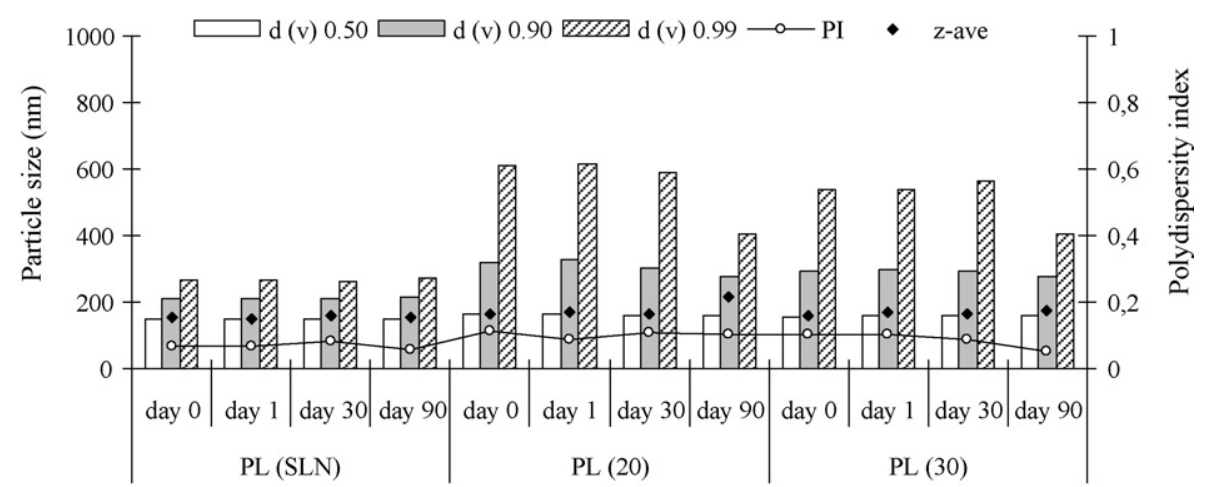

Formulation

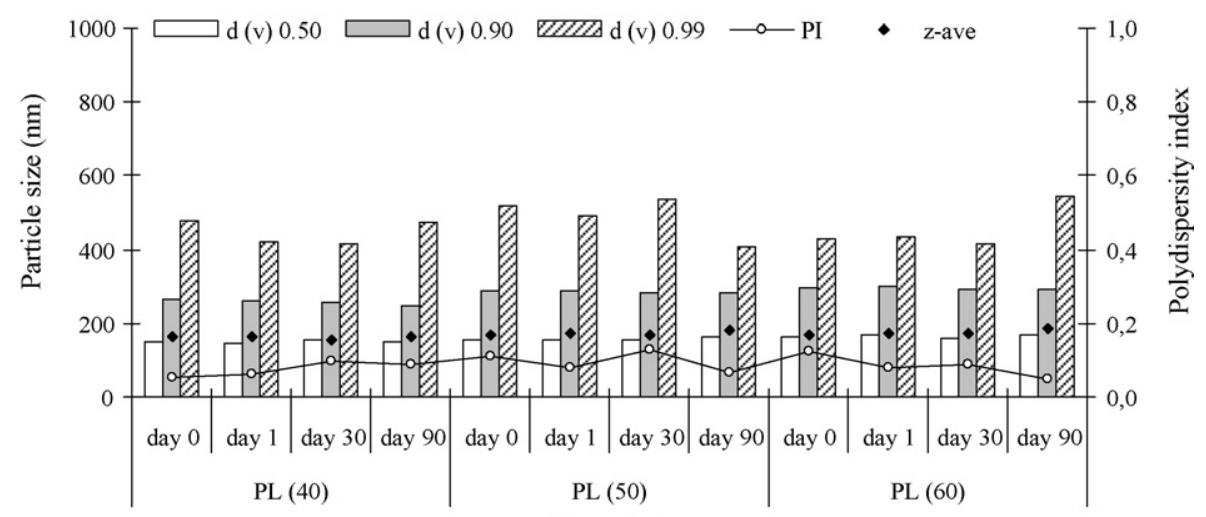

Formulation

Fig. 4. PCS data (diameter and PI) and LD data (diameters $d(v) 50 \%, d(v) 90 \%$ and $d(v) 99 \%$ ) of the formulations stabilized with PL (caprylyl/capryl glucoside) plotted as function of time $(0,1,30,90$ days $)$. 
2005). The mean diameters reflect the ability of the polyhydroxy surfactants for stabilization.

All lipid nanoparticles stabilized with PS (Plurol Stearique, polyglycerol 6-distearate) had a mean diameter of about $200 \mathrm{~nm}$, whereas the mean diameter of the samples stabilized with PL(Plantacare 810 , caprylyl/capryl glucoside) was around $170 \mathrm{~nm}$. PI values around 0.2 indicate a relatively narrow particle size distribution for the both surfactants. The oil content did not influence the mean particle size. The explanation is that during the production of NLC, the lipid mixture was in the melted state. The viscosity of melted pure cetyl palmitate and the cetyl palmitate/oil blends at this temperature is not that different. Applying the same homogenization energy, NLC containing different amounts of oil should yield the same mean particle size (Jores et al., 2004). The final size obtained may depend on various factors, such as the chemical structure of the lipids, the surfactants used, as well as their chemical interaction (Lin et al., 2007). For example, the surfactants need to be able to stabilize fast the formed droplets when they leave the homogenization gap, to avoid subsequent coalescence. Obviously the polyhydroxy surfactants are able to do this, whereas PL yields smaller particles (DLS data).

SLS and light microscopy were applied as additional methods to check for the absence of aggregates. Immediately after production a unimodal, relatively narrow particle size distribution was observed by SLS (curves not shown). The diameters $d(v) 50 \%$ were below $200 \mathrm{~nm}$, the diameters $d(v) 99 \%$ as sensitive parameter for the presence and quantity of larger particles below $600 \mathrm{~nm}$ (Figs. 3 and 4). Thus, the presence of microparticles in these samples can be excluded, to be precise: are below the detection limit. These data were in agreement with light microscopy (data not shown).

The physical stability was monitored for 90 days. In the formulations stabilized with PS the $d(v) 50 \%$ and $d(v) 99 \%$ were below $200 \mathrm{~nm}$ and $600 \mathrm{~nm}$, respectively at the production day. At day $90, d(v) 99 \%$ was still below $600 \mathrm{~nm}$ for an oil content up to $30 \%$ (w/w). Increasing the oil content from 40 to $60 \%(\mathrm{w} / \mathrm{w})$ led to an increase of $d(v) 99 \%$ (Fig. 3). Agglomerates found after 90 days in these samples were also confirmed by light microscopy. However, the degree of aggregation was small, as only the diameter $d(v) 99 \%$ is above $1 \mu \mathrm{m}$, whereas $d(v) 50 \%$ and $d(v) 90 \%$ are still below $1 \mu \mathrm{m}$. The observed slight destabilization seems to result from the expulsion of oil from the lipid matrix during the storage. Increasing the oil content from 40 to $60 \%(\mathrm{w} / \mathrm{w})$ resulted only partially into incorporation into the solid lipid matrix. This was confirmed by DSC data (cf. below). Similar results were reported for NLC based on the mixture monocaprate (solid lipid) and medium chain triglycerides which are stabilized with Polysorbate 80 as surfactant (Lin et al., 2007). In contrast to this, all lipid nanoparticle dispersions were perfectly stable when stabilized with PL (Fig. 4).

The physical stability has also been monitored by macroscopic observations. Systems remained stable with a milky-like appearance without any particle sedimentation during the storage. The absence of agglomerates after 90 days in these samples was also confirmed by light microscopy (data not shown).

\subsection{Zeta potential (ZP) analysis}

Zeta potentials of the formulations have been evaluated immediately after production (day 0 ) and after storage of 90 days. Immediately after production the zeta potentials were in the range from -30.0 to $-52.0 \mathrm{mV}$ for all samples indicating good physical stability (Fig. 5). From the literature, a minimum zeta potential of higher than $-60.0 \mathrm{mV}$ is required for excellent physical stability and of higher than $-30.0 \mathrm{mV}$ for good physical stability (Müller, 1996; Riddick, 1968).
Polyhydroxy surfactants are nonionic steric stabilizers, therefore at the first glance one would rather expect a low zeta potential. The measured zeta potential originates from the height of the Nernst potential (surface charge) and the additional charges created by adsorbed ions or surfactants or stabilizer molecules in the Stern layer. The SLN produced with solid lipid only possessed a ZP of $-30 \mathrm{mV}$ when stabilized with PS, but of $-48 \mathrm{mV}$ when stabilized with PL. It is assumed, that the major charge of the PS-stabilized nanoparticles originates from the surface, e.g. dissociated free fatty acids, some contribution potentially from salts present as impurity in the surfactant. The PL-stabilized nanoparticles possess the same particle composition (Cutina CP), but a higher ZP. This higher ZP is obviously created by the different type of stabilizer. The PL has one hydrophobic anchor to be embedded in the surface, and one long hydrophilic tail composed of glucose molecules penetrating into the water phase. The glucoses will strongly interact with water, e.g. attracting potentially negatively charged hydroxyl ions. The PS has two hydrophobic anchors located at the end of the molecule, polyglycerol in the center. Assuming both lipophilic parts anchored into the particle surface, the molecule will have a loop structure on the surface. These differences are considered as cause for the different ZP values.

With increasing oil content in the particle matrix, the ZP of the PL-stabilized nanoparticles stays unchanged. It stays also unchanged during the 90 days of storage, being the reason of the observed perfect physical stability (cf. Section 3.2).

Interestingly, the ZP of the PS-stabilized nanoparticles increases with increasing oil content form $-32 \mathrm{mV}$ (no oil) to about $-50 \mathrm{mV}$ (60\% oil). Assuming an unchanged situation for the stabilizer layer on the surface, this increase in the ZP can only originate from an increase of the surface potential. Obviously there are more charges on the surface, potential source are free fatty acids contained in the solid lipid but also in the oil. The oil nanocompartments of NLC can be embedded into the solid lipid matrix (Müller et al., 2002b) or to be localised at the surface of solid platelets and the surfactant layer (Jores et al., 2005). Therefore this increase in zeta potential can be the result of changes on the NLC surface. These changes did not occur with PL, this supports the theory that the surfactants used also affect the structure of the lipid particle matrix, including localization of oil.

During the storage of 90 days, the zeta potential of the PSstabilized NLC decreased for an oil content of 40-60\%. Obviously there must be changes in the composition of particle matrix and related surface composition and charge. Without additional investigations, discussing potential reasons would be speculative. Further mechanistic investigations are necessary for a better understanding of this effect, because the structural change is obviously stabilizer-related, as it does not occur with PL. The understanding of such influences would allow a more controlled selection of optimal stabilizers for lipid nanoparticle dispersions in the future.

However, in these PS-stabilized formulations the zeta potential is still higher than $-30.0 \mathrm{mV}$ after 90 days of storage. Hence from ZP theory, the values are in the range for stable dispersions. Therefore the slight instability observed in these samples with $40-60 \%$ oil content might be due to destabilizing effects not accessible by a zeta potential measurement. It should be pointed out, that the increase in the diameter $90 \%$ is very low, not affecting the use of these NLC in dermal formulations.

\subsection{Influence of hydrophilic-lipophilic-balance (HLB) of the stabilizer on size and stability}

The particle size and the physical stability of a disperse system are influenced by many parameters, e.g. type and concentration of the stabilizer. Up today it is not possible to fully predict the size and the physical stability without practical investigations. However, 


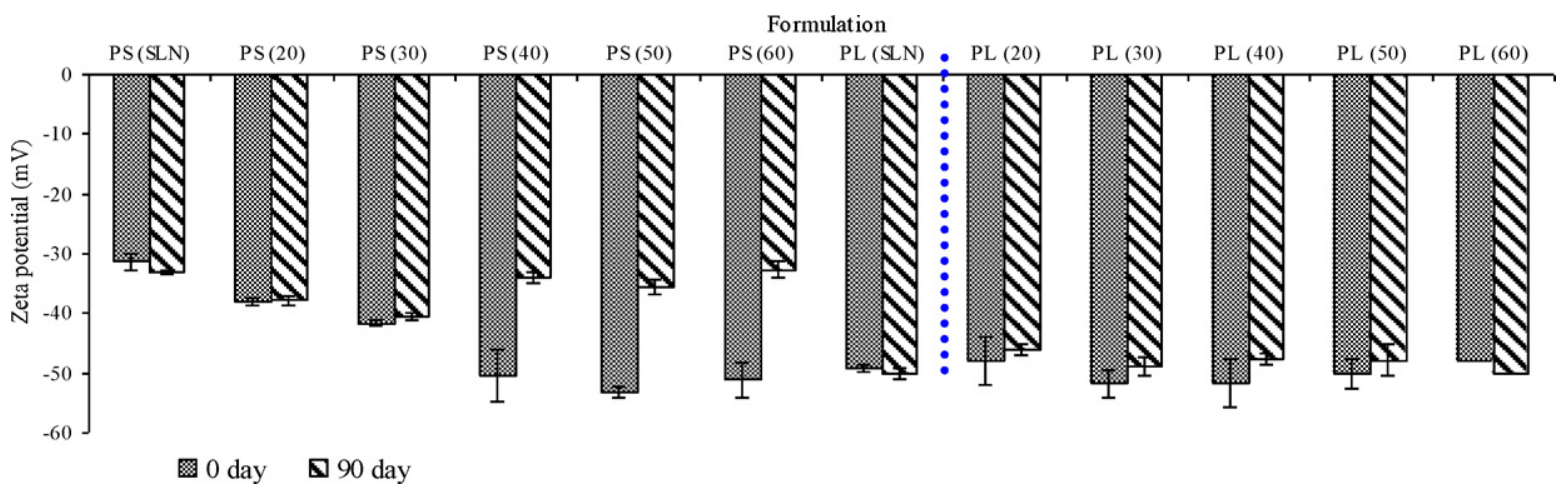

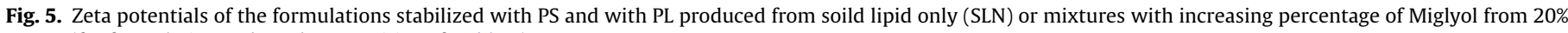
to $60 \%$ (for formulation code and composition cf. Table 3 ).

parameters e.g. zeta potential and polydispersity are important tools, as they can indicate good stability at an early stage of the development. Nevertheless, in the field of emulsion formulation, especially in the cosmetic field, another possibility to obtain finely dispersed and physically stable emulsions is the estimation of the "required HLB". This means, that the HLB of the stabilizer should match the required HLB of the inner phase as close as possible. Such a required HLB can be either calculated using Grifinth equation or by assessing the values provided by manufactures. For the lipids used in this study the required HLB is 10 for the solid lipid and 5 for the liquid lipid. Hence with an increasing amount of liquid lipid the HLB decreases. The surfactants used in this study possess different HLB values. The HLB of PS is 9-10 and the HLB of PL is 15-16 (cf. Table 1). Thus, from theory, best results (smallest size, highest stability) would be expected for the SLN and the NLC containing 20\% oil stabilized with PS (HLB 9-10), respectively. Indeed, these formulations are physically stable, whereas the NLC containing more oil, e.g. 40-60\% (required HLB of these formulations is 8-7) are less stable (cf. Fig. 4). However, the size of the SLN stabilized with PL is smaller than the size of the SLN stabilized with PS, even though the HLB of PL is much higher and thus not as close to the required HLB of the lipid phase as PS (cf. Figs. 3 and 4). Also for the NLC with high oil content the lower and more close HLB of PS was less sufficient in stabilizing the NLC, whereas the high HLB surfactant PL, could stabilize all lipid nanoparticle systems investigated sufficiently (cf. Figs. 3 and 4). Thus, in conclusion the "required HLB" theory cannot be used to explain the differences between the two stabilizers investigated. In fact, results prove that the development of a finely dispersed and physically stable lipid nanoparticle system is very complex, not yet fully understood, and cannot only be reached by simply using the "required HLB" method alone.

\subsection{Differential scanning calorimetry investigations}

Table 4 gives an overview of the DSC data of SLN and of NLC dispersions, which have been stored for a period of 90 days at controlled room temperature. After this time, an equilibrium condition can be assumed. The nanonized cetyl palmitate is distinctly below the melting temperature of the bulk material (melting point depression by about $4^{\circ} \mathrm{C}$ ). This phenomenon is described by the Gibbs-Thompson effect, i.e. the larger ratio of specific surface area to volume of particle with a smaller size when compared to bulk material (Perez, 2005). A shift of the melting point to lower temperatures with decreasing mean particle size was also observed for Dynasan ${ }^{\circledR} 116$ dispersions stabilized with Tyloxapol ${ }^{\circledR}$ (Souto, 2005). Beside the small particle size, the potential influence of surfactants should not to be neglected. Depending on the lipophilicity, the sur- factants partition between water phase, interface and the lipid phase. Surfactant in the lipid phase can distort crystallization and affect the melting temperature. Interestingly, the melting point is the same for SLN stabilized with PS or with PL, i.e. for nanoparticles made from solid lipid only. The same applies for the NLC regarding melting point, but also the onset temperature.

However, the situation is different for the recrystallisation index RI (cf. Section 2.2.5). For the SLN, the RI is reduced to about $60 \%$ when stabilized with PS, but reduced to only $32 \%$ in case of PL as stabilizer (Table 4). The same is valid for all NLC. The ones stabilized with PL possess much less crystallinity. PS stabilized NLC with $50 \%$ oil in the matrix (PS (50)) have a RI of $12 \%$, the corresponding PL (50) dispersion shows no melting event any more, i.e. the dispersion is a supercooled nanoemulsion (Table 4 and Fig. 6). At $60 \%$ oil in the particle matrix, both formulations are not solid any more. This shows firstly the strong influence of the type of surfactant on the crystallinity, and secondly is a nice example for differences in re-crystallization between bulk and nanomaterial.

The decreasing of melting peak and melting enthalpy, i.e. distortion of the crystalline lattice in NLC after oil addition, is identical to the distortion of crystalline structure of lipid blends in the bulk state, but more pronounced in the nano dimension. This less ordered crystalline lipid matrix of NLC is favourable for encapsulating more active (higher loading).

The differences in the shapes of DSC curves in Fig. 6 (PS versus $\mathrm{PL}$ ) show that the internal structure of lipid nanoparticles is influenced by the type of polyhydroxy surfactants used. It is known that the surfactants can have impact on the resulting structure of dispersed phases. In o/w emulsions containing emulsifiers with long saturated fatty acid chains, the increased crystallization tendency of the dispersed phase has been correlated with the formation of emulsifiers templates in the oil droplets. Investigations on tripalmitin nanoparticles indicated that rigid long saturated alkyl chains of surfactants (Polysorbate 80 ) are necessary to induce crystallization. PS (polyglycerol 6-distearate) the surfactant containing long saturated alkyl chain (solid surfactant!) might initiate crystallization of lipid matrix of nanoparticles, explaining the higher RI values. Due to the affinity of polyglycerol 6-distearate to cetyl palmitate, a small portion of the surfactant molecules localize in the lipid phase (partitioning coefficient) and thus promote recrystallization of the lipid. A part of the stabilizer is included into the lipid matrix of nanoparticles, stabilizers are not only attached onto the surface. Similar results have been obtained for SLN containing tripalmitate as solid lipid and lecithin as emulsifier (Ahlin et al., 2000).

In contrast, PL (caprylyl/capryl glucoside) with short hydrophobic alkyl chains (liquid surfactant!) is less hydrophobic, and thus 
Table 4

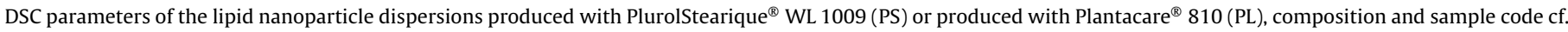
Table 3.

\begin{tabular}{|c|c|c|c|c|c|}
\hline Formulation & Melting temperature $\left[{ }^{\circ} \mathrm{C}\right]$ & Onset temperature $\left[{ }^{\circ} \mathrm{C}\right]$ & Enthalpy [J/g] & RI [\%] & Integral [mJ] \\
\hline PS (SLN) & 48.80 & 43.80 & 13.3 & 60.33 & 190.55 \\
\hline PS (20) & 47.97 & 43.61 & 8.15 & 36.97 & 133.40 \\
\hline PS (30) & 46.98 & 43.90 & 4.92 & 22.32 & 80.56 \\
\hline PS (40) & 45.77 & 42.73 & 4.32 & 19.59 & 86.52 \\
\hline PS (50) & 45.55 & 42.18 & 2.70 & 12.24 & 46.89 \\
\hline PS (60) & No melting event & & & & \\
\hline PL (SLN) & 48.79 & 44.6 & 7.12 & 32.30 & 130.89 \\
\hline $\mathrm{PL}(20)$ & 47.35 & 42.42 & 5.16 & 23.40 & 88.33 \\
\hline PL (30) & 46.78 & 42.06 & 3.83 & 17.37 & 77.35 \\
\hline PL (40) & 47.15 & 45.30 & 0.46 & 2.08 & 9.37 \\
\hline PL (50) & No melting event & & & & \\
\hline PL (60) & No melting event & & & & \\
\hline
\end{tabular}
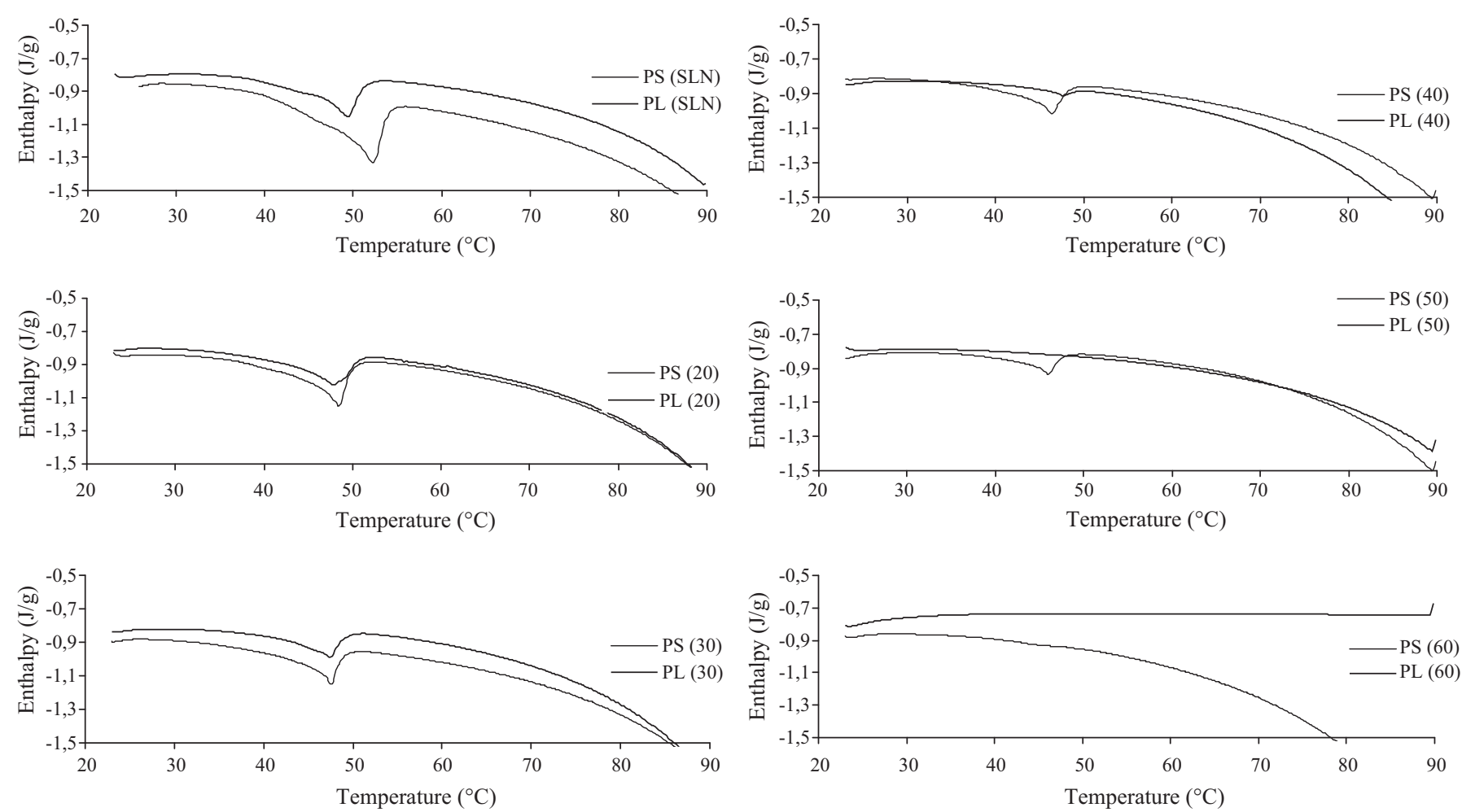

Fig. 6. DSC heating scans of the aqueuos lipid nanoparticle dispersions at day 90 (scan from 20 to $90{ }^{\circ} \mathrm{C}$, scan rate $10 \mathrm{~K} \mathrm{~min}{ }^{-1}$, formulation code cf. Table 3 ).

might locate less in the lipid phase, as it is too mobile in order to induce crystallization of lipid in NLC containing a high oil content. This surfactant probably forms rather a more "fluid", less rigid membrane around nanodroplets with a low microviscosity. Therefore, polyglycerol 6-distearate with a "solid lipid tail" in the structure leads to a different interaction with lipid matrix molecules, than the "liquid lipid tail" surfactant (caprylyl/capryl glucoside).

The onset and the melting process of SLN and NLC occur at the temperature above $40^{\circ} \mathrm{C}$. This is especially important for lipid nanoparticles for dermal application of pharmaceutical and cosmetic actives. The particles should remain in the solid state when applied.

The PS stabilized NLC possess a higher crystallinity than the ones with PL, this can cause phase separation, e.g. drug expulsion but also potentially expulsion/separation of oil from solid lipid. Thus could lead to changes in the composition of the surface, and related changes in Nernst and zeta potential. The zeta potentials decreased during storage. Such phenomena might also promote bridging between particles.

\section{Conclusions}

Both polyhydroxy surfactants in the concentration of $1 \%(w / w)$ led to SLN and NLC with a mean diameter of about $200 \mathrm{~nm}$. SLN and NLC with an oil content up to $30 \%(\mathrm{w} / \mathrm{w}$ ) were physically stable over the investigated period of 90 days. However, increasing the oil content to $40 \%$ and higher led to a slight increase in larger particles $(d(v) 0.99)$ in NLC stabilized with PS (polyglycerol 6-distearate). This effect was attributed to the higher crystallinity and related expulsion effects. The formulations stabilized with PL (caprylyl/capryl glucoside) were perfectly stable at each oil content. However, NLC with an oil content of $50 \%$ or higher and of $60 \%$ (PS and PL, respectively) were not solid anymore - in contrast to the bulk lipid blends. This shows the limitation in nanostructuring of NLC by oil addition, and the different re-crystallization properties in the nanodimension.

It can be concluded that the crystallization tendency of the particles increases with the length of the saturated hydrophobic chain of polyhydroxy surfactant. These observations suggest that the crystallization promoting effect of polyhydroxy surfactants is caused by 
surfactant structure but also the interaction of the surfactant and lipid matrix molecules.

In overall conclusion polyhydroxy surfactants proved suitable for the stabilization of SLN and NLC dispersions, they are therefore an interesting newly applied class of stabilizers for future dermal products. They can influence the crystalline state of the lipid matrix in nanoparticles and for a successful formulation development, this parameter needs to be investigated. Furthermore, it might be a helpful tool to develop NLC with a "tailor-made" crystalline state, resulting loading capacity and release profile in the future.

\section{References}

Ahlin, P., Kristl, J., Sentjurc, M., Strancar, J., Pecar, S., 2000. Influence of spin probe structure on its distribution in SLN dispersions. Int. J. Pharm. 196, 241-244.

Attama, A.A., Schicke, B.C., Paepenmüller, T., Müller-Goymann, C.C., 2007. Solid lipid nanodispersions containing mixed lipid core and a polar heterolipid: characterization. Eur. J. Pharm. Biopharm. 67, 48-57.

Blasi, P., Giovagnoli, S., Schoubben, A., Ricci, M., Rossi, C., 2007. Solid lipid nanoparticles for targeted brain drug delivery. Adv. Drug Deliv. Rev. 59, 454-477.

Bunjes, H., Koch, M.H., Westesen, K., 2002. Effects of surfactants on the crystallization and polymorphism of lipid nanoparticles. Prog. Colloid Polym. Sci. 121, 7-10.

Bunjes, H., Koch, M.H., Westesen, K., 2003. Influence of emulsifiers on the crystallization of solid lipid nanoparticles. J. Pharm. Sci. 92, 1509-1520.

Cosmetic Ingredient Reviews, 1999. Final report on the safety assessment of PEG-2, $-3,-5,-10,-15$, and -20 cocamine. Int. J. Toxicol. 18, 43-50.

Freitas, C., Müller, R.H., 1999. Correlation between long-term stability of solid lipid nanoparticles (SLN) and crystallinity of the lipid phase. Eur. J. Pharm. Biopharm. 47, 125-132.

Garti, N., Yano, J., 2001. The roles of emulsifiers in fat crystallization. In: Sato, K., Garti, S. (Eds.), Crystallization Processes in Fats and Lipid Systems. Marcel Dekker, New York, pp. 211-250.

Johnson Jr., W., 2001. Final report on the safety assessment of PEG-25 propylene glycol stearate, PEG-75 propylene glycol stearate, PEG-120 propylene glycol stearate, PEG-10 propylene glycol PEG-8 propylene glycol cocoate, and PEG-55 propylene glycol oleate. Int. J. Toxicol. 20 Suppl. 4, 13-26.

Jores, K., Haberland, A., Wartewig, S., Mäder, K., Mehnert, W., 2005. Solid lipid nanoparticles (SLN) and oil-loaded SLN studied by spectrofluorometry and Raman spectroscopy. Pharm. Res. 22, 1887-1897.

Jores, K., Mehnert, W., Drechsler, M., Bunjes, H., Johann, C., Mäder, K., 2004. Investigations on the structure of solid lipid nanoparticles (SLN) and oil-loaded solid lipid nanoparticles by photon correlation spectroscopy, field-flow fractionation and transmission electron microscopy. J. Control. Rel. 95, 217-227.

Joshi, M.D., Müller, R.H., 2009. Lipid nanoparticles for parenteral delivery of actives. Eur. J. Pharm. Biopharm. 71, 161-172.

Keck, C.M., Müller, R.H., 2008. Size analysis of submicron particles by laser diffractometry-90\% of the published measurements are false. Int. J. Pharm. 355, 150-163.

Lanigan, R.S., Yamarik, T.A., 2001. Final report on the safety assessment of PEG-6, -8, and -20 sorbitan beeswax. Int. J. Toxicol. 20, 27-38.

Lin, X., Li, X., Zheng, L., Yu, L., Zhang, Q., Liu, W., 2007. Preparation and characterization of monocaprate nanostructured lipid carriers. Colloid Surf. A: Physicochem. Eng. Aspects 311, 106-111.

Liu, J., Gong, T., Fu, H., Wang, C., Wang, X., Chen, Q., Zhang, Q., He, Q., Zhang, Z., 2008. Solid lipid nanoparticles for pulmonary delivery of insulin. Int. J. Pharm. 356, 333-344.

Lombardi Borgia, S., Regehly, M., Sivaramakrishnan, R., Mehnert, W., Korting, H.C., Danker, K., Röder, B., Kramer, K.D., Schäfer-Korting, M., 2005. Lipid nanoparticles for skin penetration enhancement-correlation to drug localization within the particle matrix as determined by fluorescence and parelectric spectroscopy. J. Control. Rel. 110, 151-163.

Lombardi Borgia, S., Schlupp, P., Mehnert, W., Schäfer-Korting, M., 2007. In vitro skin absorption and drug release-a comparison of six commercial prednicarbate preparations for topical use. Eur. J. Pharm. Biopharm..

Lucks, J.S., Müller, R.H., 1996. Medication vehicles made of solid lipid particle (solid lipid Nanospheres-SLN), European Patent EP0000605497.
Mehnert, W., Mäder, K., 2001. Solid lipid nanoparticles: production, characterization and applications. Adv. Drug Deliv. Rev. 47, 165-196.

Muchow, M., Maincent, P., Müller, R.H., 2008. Lipid nanoparticles with a solid matrix (SLN, NLC LDC) for oral drug delivery. Drug Dev. Ind. Pharm. 34 1394-1405.

Müller, R.H., 1996. Zetapotential und Partikeladung in der Laborpraxis. Wissenschaftliche Verlagsgeselschaft mbH. Suttgart.

Müller, R.H., Jenning, V., Mäder, K., Lippacher, A., 2000a. Lipid Particles on the Basis of Mixtures of Liquid and Solid Lipids and the Method for Producing same, PCT/EP2000/004112. PharmaSol GmbH, Germany.

Müller, R.H., Mäder, K., Gohla, S., 2000b. Solid lipid nanoparticles (SLN) for controlled drug delivery-a review of the state of the art. Eur. J. Pharm. Biopharm. 50, 161-177.

Müller, R.H., Mehnert, W., Lucks, J.S., Schwarz, C., zur Mühlen, A., Weyhers H., Freitas, C., Rühl, D., 1995. Solid lipid nanoparticles (SLN)-an alternative colloidal carrier system for controlled drug delivery. Eur. J. Pharm. Biopharm. 41, 62-69.

Müller, R.H., Petersen, R.D., Hommoss, A., Pardeike, J., 2007. Nanostructured lipid carriers (NLC) in cosmetic dermal products. Adv. Drug Deliv. Rev. 59, 522-530.

Müller, R.H., Radtke, M., Wissing, S.A., 2002a. Nanostructured lipid matrices for improved microencapsulation of drugs. Int. J. Pharm. 242, 121-128.

Müller, R.H., Radtke, M., Wissing, S.A., 2002b. Solid lipid nanoparticles (SLN) and nanostructured lipid carriers (NLC) in cosmetic and dermatological preparations. Adv. Drug Deliv. Rev. 54, S131-155.

Müller, R.H., Runge, S., Ravelli, V., Mehnert, W., Thünemann, A.F., Souto, E.B., 2006 Oral bioavailability of cyclosporine: solid lipid nanoparticles (SLN) versus drug nanocrystals. Int. J. Pharm. 317, 82-89.

Pardeike, J., Hommoss, A., Müller, R.H., 2009. Lipid nanoparticles (SLN, NLC) in cosmetic and pharmaceutical dermal products. Int. J. Pharm. 366, 170-184.

Perez, M., 2005. Gibbs-Thomson effects in phase transformations. Scripta Mater. 52 709-712.

Rawle, A. Basic principles of particle size analysis. Online article, www.malvern.com.

Riddick, T.M., 1968. Control of Colloid Stability through Zeta Potential. Zeta-Meter Inc. via Livingston Publishing Company, Wynnewood.

Santos Maia, C., Mehnert, W., Schaller, M., Korting, H.C., Gysler, A., Haberland, A., Schäfer-Korting, M., 2002. Drug targeting by solid lipid nanoparticles for dermal use. J. Drug Target. 10, 489-495.

Sarmento, B., Martins, S., Ferreira, D., Souto, E.B., 2007. Oral insulin delivery by means of solid lipid nanoparticles. Int. J. Nanomed. 2, 743-749.

Saupe, A., Wissing, S.A., Lenk, A., Schmidt, C., Müller, R.H., 2005. Solid lipid nanoparticles (SLN) and nanostructured lipid carriers (NLC)-structural investigations on two different carrier systems. Biomed. Mater. Eng. 15, 393-402.

Siekmann, B., 1994. Untersuchungen zur Herstellung und zum Rekristallisationsverhalten schmelzemulgierter intravenös applizierbarerGlyceridnanopartikel. Ph.D. Thesis, Technische Universität Carlo Wilhelmina Braunschweig.

Siekmann, B., Westesen, K., 1994a. Melt-homogenized solid lipid nanoparticles stabilized by the nonionic surfactant tyloxapol. I. Preparation and particle size determination. Pharm. Pharmacol. Lett. 3, 194-197.

Siekmann, B., Westesen, K., 1994b. Melt-homogenized solid lipid nanoparticles stabilized by the nonionic surfactant tyloxapol. II. Physicochemical characterization and lyophilisation. Pharm. Pharmacol. Lett. 3, 225-228.

Söderman, O., Johansson, I., 1999. Polyhydroxyl-based surfactants and their physicochemical properties and applications. Curr. Opin. Colloid Interface Sci. 4, 391-401.

Souto, E.B., 2005. SLN and NLC for Topical Delivery of Antifungals. Ph.D. Thesis, Freie Universität Berlin.

Souto, E.B., Wissing, S.A., Barbosa, C.M., Müller, R.H., 2004. Development of a controlled release formulation based on SLN and NLC for topical clotrimazole delivery. Int. J. Pharm. 278, 71-77.

Stecova, J., Mehnert, W., Blaschke, T., Kleuser, B., Sivaramakrishnan, R., Zouboulis C.C., Seltmann, H., Korting, H.C., Kramer, K.D., Schäfer-Korting, M., 2007. Cyproterone acetate loading to lipid nanoparticles for topical acne treatment: particle characterisation and skin uptake. Pharm. Res. 24, 991-1000.

Tadros, T.F., 2005. Applied Surfactants-Principles and Applications. Wiley VHC, Weinheim.

Ugazio, E., Cavalli, R., Gasco, M.R., 2002. Incorporation of cyclosporin A in solid lipid nanoparticles (SLN). Int. J. Pharm. 241, 341-344

Westesen, K., Siekmann, B., Koch, M.H., 1993. Investigations on the physical state of lipid nanoparticles by synchron X-ray diffraction. Int. J. Pharm. 93, 189-199.

Wissing, S.A., Kayser, O., Müller, R.H., 2004. Solid lipid nanoparticles for parenteral drug delivery. Adv. Drug Deliv. Rev. 56, 1257-1272. 